\title{
BMJ Open Risk of neurodegenerative dementia in asthma patients: a nested case-control study using a national sample cohort
}

\author{
So Young Kim, ${ }^{1}$ Chanyang Min, ${ }^{2}$ Dong Jun Oh, ${ }^{3}$ Hyo Geun $\mathrm{Choi}^{4}$
}

To cite: Kim SY, Min C, Oh DJ, et al. Risk of neurodegenerative dementia in asthma patients: a nested case-control study using a national sample cohort. BMJ Open 2019;9:e030227. doi:10.1136/ bmjopen-2019-030227

- Prepublication history and additional material for this paper are available online. To view please visit the journal (http:// dx.doi.org/10.1136/bmjopen2019-030227).

Received 05 March 2019 Revised 29 August 2019 Accepted 06 September 2019

Check for updates

(C) Author(s) (or their employer(s)) 2019. Re-use permitted under CC BY-NC. No commercial re-use. See rights and permissions. Published by BMJ.

\section{${ }^{1}$ Department of}

Otorhinolaryngology-Head \& Neck Surgery, CHA University, Seongnam, Korea (the Republic of)

${ }^{2}$ Hallym Data Science Laboratory, Hallym University College of Medicine, Anyang-si, Korea (the Republic of)

${ }^{3}$ Asan Medical Center, University of Ulsan College of Medicine,

Seoul-si, Korea (the Republic of) ${ }^{4}$ Department of

Otorhinolaryngology Head \&

Neck Surgery, Hallym University, Anyang-si, Korea (the Republic of)

Correspondence to

Dr Hyo Geun Choi;

pupen@naver.com

\section{ABSTRACT}

Objectives This study investigated the risk of neurodegenerative dementia following asthma.

Design A nested case-control study

Setting The $\geq 60$-year-old population was selected from the Korean Health Insurance Review and Assessment Service - National Sample Cohort from 2002 to 2013. Participants and interventions The 11442 dementia cases were matched with 45768 control cases for age, sex, income, region of residence, hypertension, diabetes and dyslipidaemia. Asthma was classified using International Classification of Disease-10 (ICD-10) codes (J45 and J46) and medication history. Dementia was identified based on ICD-10 codes (G30 and F00).

Primary and secondary outcome measures The ORs of a previous history of asthma in patients with dementia were analysed using conditional logistic regression analysis stratified for age, sex, income, region of residence, hypertension, diabetes and dyslipidaemia. Subgroup analysis was performed according to age and sex.

Results Overall, 22.6\% (2587/11 442) and 22.3\% (10 $229 / 45768$ ) of the cases in the dementia and control groups, respectively, had a previous history of asthma. The OR for asthma in the dementia group was not higher than that in the control group (adjusted $\mathrm{OR}=0.97,95 \%$ Cl 0.92 to $1.02, p=0.207$ ). All age and sex subgroups demonstrated consistent results.

Conclusions Asthma was not related to an increased risk of dementia.

\section{INTRODUCTION}

Dementia is an age-related neurodegenerative disease on a spectrum from mild cognitive impairment to a full-blown dementia state. Because of an ageing society and improved life expectancy by virtue of advanced medical care, an increasing number of people suffer from dementia. The incidence of dementia is estimated to be approximately 14.3 per 1000 person-years in men and 17.0 per 1000 person-years in women aged $\geq 50$ years old in Western countries. ${ }^{1}$ In Korea, the prevalence of dementia was estimated to be approximately 6\%-10\%. ${ }^{2}$ Because older adults have a higher number of comorbidities such as cardiovascular and metabolic diseases, the

\section{Strengths and limitations of this study}

This study was based on a large population of older adults considering the high prevalence of dementia in this age group.

- Moreover, the potential biases between the dementia and control groups were minimised by matching medical histories as well as demographic factors.

- Although ICD-10 codes are based on the diagnosis of physicians, they lack information on the severity of disease and treatment history.

impact of these systemic disorders on neurodegenerative changes is anticipated to grow. Consistent with these findings, numerous studies have reported associations of various chronic diseases, including hypertension, diabetes, dyslipidaemia, coronary heart disease and depression, with dementia. ${ }^{3-6}$

Asthma is a chronic airway disease defined by typical pulmonary dysfunction and airway symptoms. The prevalence of asthma has been reported to be approximately $2 \%-20 \%$ with both increasing and decreasing tendencies, depending on the ethnic group studied. ${ }^{7}$ The incidence of asthma in Korea was reported to be stable at approximately 6.07 per 1000 person-years. $^{8}$ In addition to the well-known type 2 helper $\mathrm{T}$ cell-related asthma, various endotypes of asthma associated with multiple pathophysiological mechanisms have been described. ${ }^{9}$ Due to the many contributors, asthma was reported to be associated with many chronic diseases, such as hypertension, diabetes, dyslipidaemia, coronary heart disease and depression. ${ }^{10-13}$ Dementia has also been proposed to be related to asthma. A national population study reported a 2.17-fold higher risk of dementia in asthma patients $>45$ years old $(95 \%$ CIs 1.87 to 2.52). ${ }^{14}$ Another national population study showed a 1.27-fold higher risk of dementia in asthma patients $\geq 20$ years old $(95 \%$ CI 1.15 to 1.41$).{ }^{15}$ The authors of that study hypothesised that the common pathophysiology of 
inflammation, immune dysfunction and cardiovascular compromise might link asthma to dementia. However, both studies used young and middle-aged adult populations. In addition, control groups were matched with asthma patients for age and sex only; thus, comorbidities such as hypertension, diabetes and dyslipidaemia were higher in the asthmatic groups. Adjusting for these comorbidities might influence the differences in the risk of dementia between the asthma and control groups.

Therefore, it was questioned whether asthma increases the risk of dementia in the older population, irrespective of other comorbid conditions. We hypothesised that the risk of dementia in patients with asthma may have been overestimated in previous studies. To test this hypothesis, patients with dementia were investigated for a previous history of asthma compared with the control group matched for age, sex, income, region of residence and medical histories of hypertension, diabetes and dyslipidaemia.

\section{MATERIALS AND METHODS \\ Participant selection}

Out of 1125691 cases with 114369638 medical claim codes, we included cases with a diagnosis of dementia from 2002 to 2013 ( $n=13102)$. Cases were also classified as dementia if a diagnosis of Alzheimer's disease (ICD-10 code: G30) or dementia in Alzheimer's disease (F00) had been made. For accurate diagnosis, we selected only cases who were treated $\geq 2$ times. We described the reliability of the diagnosis of dementia in the online supplementary material $\mathrm{S} 1$. The control cases who were never diagnosed with dementia were extracted from this cohort of 1112589 participants from 2002 to 2013.

We included cases who were diagnosed with asthma (ICD-10: J45) or status asthmaticus (J46). Among them, we selected participants who were treated $\geq 2$ times and who were treated with a corticosteroid, steroid inhaler, Long-acting muscarinic antagonists (LAMA), leukotriene receptor antagonists (LTRA) or Xantine $(n=230764)$. This method has been modified from a previous study. ${ }^{16}$

The dementia cases were matched at a 1:4 ratio with patients (control group) in this cohort who had never been treated for dementia from 2002 to 2013. The control group was selected from the original population $(n=1112589)$. These subjects were matched for age, sex, income, region of residence and medical history (hypertension, diabetes and dyslipidaemia). To prevent a selection bias when selecting the matched cases, the control group cases were sorted using a random number order, and they were then selected from top to bottom. The matched control cases were assumed to be involved at the same time as each matched dementia case (index date). Therefore, control group subjects who died before the index date were excluded. Dementia cases for whom we could not identify enough matched cases were excluded $(\mathrm{n}=1148)$. We also excluded cases under 60 years old $(n=512)$. Finally, 1:4 matching resulted in the inclusion of 11442 dementia cases and 45768 control cases (figure 1). However, the cases were not matched for ischaemic heart disease, cerebral stroke and depression because strict matching would have increased the number of excluded study cases due to a lack of control cases. After matching, we analysed the cases' previous histories of asthma in both the dementia and control groups.

\section{Variables}

The cases were grouped by age using 5 -year intervals as follows: 60-64, 65-69, 70-74... and 85+ years old. Age was defined as age at the onset of dementia, and six age groups were designated. The income groups were initially divided into 41 classes ( 1 health aid class, 20 self-employed

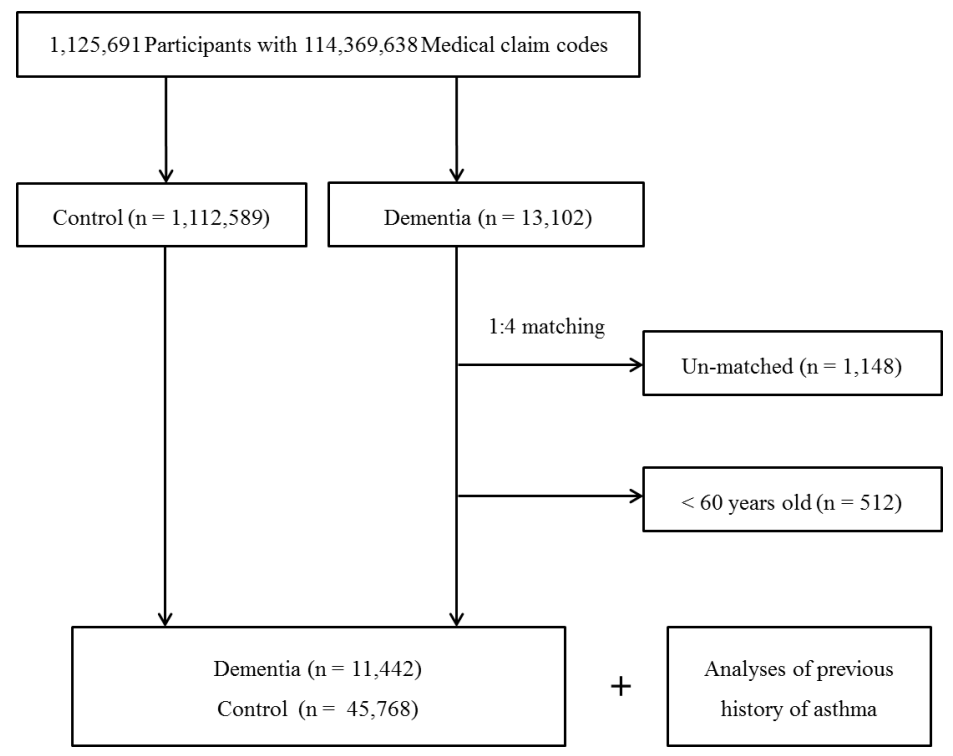

Figure 1 Schematic illustration of the participant selection process that was used in the present study. Of a total of 1125691 cases, 11442 dementia cases were matched with 45768 control cases with respect to age, sex, income, region of residence and medical history. 
health insurance classes and 20 employed health insurance classes). These groups were recategorised into five classes (class 1 (lowest income) - class 5 (highest income)). Region of residence was divided into 16 areas according to administrative district. These regions were regrouped into urban (Seoul, Busan, Daegu, Incheon, Gwangju, Daejeon and Ulsan) and rural (Gyeonggi, Gangwon, Chungcheongbuk, Chungcheongnam, Jeollabuk, Jeollanam, Gyeongsangbuk, Gyeongsangnam and Jeju) areas.

The medical histories of the cases were evaluated using ICD-10 codes. For the accuracy of diagnosis, hypertension (I10 and I15), diabetes (E10-E14) and dyslipidaemia (E78) were assessed if the cases were treated $\geq 2$ times. Ischaemic heart disease (I24 and I25) and cerebral stroke (I60-I66) were assessed if the cases were treated $\geq 1$ time. Depression was defined using ICD-10 codes F31 (bipolar affective disorder) through F39 (unspecified mood disorder) recorded by a psychiatrist $\geq 2$ times. Chronic obstructive pulmonary disease (COPD) was determined by $\mathrm{J} 43$ (emphysema) through J44 (other COPD) and treatment with Short-Acting Beta-Agonists (SABA), longacting beta agonists (LABA), Long-acting muscarinic antagonists (LAMA) or corticosteroids. ${ }^{17}$

\section{Statistical analyses}

A $\chi^{2}$ test was used to compare the rate of general characteristics between the dementia and control groups.

To analyse the OR of asthma (dependent variable) with dementia (independent variable), conditional logistic regression analysis was used. In these analyses, crude (simple) and adjusted (ischaemic heart disease, cerebral stroke, depression and COPD) models were used, and 95\% CIs were calculated. In these analyses, groups were stratified by age, sex, income, region of residence, hypertension, diabetes and dyslipidaemia.

For the subgroup analyses, we divided the cases by age and sex (men $<80$ years old, women $<80$ years old, men $\geq 80$ years old and women $\geq 80$ years old). The dividing point of the age groups was determined by median values.

Two-tailed analyses were conducted, and $\mathrm{p}$ values $<0.05$ were considered to indicate significance. The results were analysed using SPSS V.22.0.

\section{Patients and public involvement statement}

This national cohort study used data from the Korean National Health Insurance Service-National Sample Cohort (NHIS-NSC). The detailed description of these data was described in our previous studies. ${ }^{18} 19$ No patients were involved in the development of the research question or the design of the study. We have no plan to disseminate the results to the cases. Because the NHIS-NSC data are based on national health claim codes, releasing the data by the researcher is not allowed legally. All data are available from the database of National Health Insurance Sharing Service (NHISS) (https://nhiss.nhis.or.kr/).

NHISS allows all of these data for any researcher who promises to follow the research ethics with some cost. If

\begin{tabular}{|c|c|c|c|}
\hline \multirow[b]{2}{*}{ Characteristics } & \multicolumn{3}{|c|}{ Total participants } \\
\hline & $\begin{array}{l}\text { Dementia (n, } \\
\%)\end{array}$ & $\begin{array}{l}\text { Control group } \\
(n, \%)\end{array}$ & $P$ value \\
\hline Age (years old) & & & 1.000 \\
\hline $60-64$ & $580(5.1)$ & $2320(5.1)$ & \\
\hline $65-69$ & 1289 (11.3) & 5156 (11.3) & \\
\hline $70-74$ & 2325 (20.3) & 9300 (20.3) & \\
\hline $75-79$ & 2979 (26.0) & 11916 (26.0) & \\
\hline $80-84$ & 2703 (23.6) & $10812(23.6)$ & \\
\hline $85+$ & 1566 (13.7) & $6264(13.7)$ & \\
\hline Sex & & & 1.000 \\
\hline Male & 3663 (32.0) & 14652 (32.0) & \\
\hline Female & 7779 (68.0) & $31116(68.0)$ & \\
\hline Income & & & 1.000 \\
\hline 1 (lowest) & $2866(25.0)$ & $11464(25.0)$ & \\
\hline 2 & $1034(9.0)$ & $4136(9.0)$ & \\
\hline 3 & $1374(12.0)$ & 5496 (12.0) & \\
\hline 4 & $1884(16.5)$ & 7536 (16.5) & \\
\hline 5 (highest) & 4284 (37.4) & $17136(37.4)$ & \\
\hline Region of residence & & & 1.000 \\
\hline Urban & $4623(40.4)$ & $18492(40.4)$ & \\
\hline Rural & 6819 (59.6) & 27276 (59.6) & \\
\hline Hypertension & 8316 (72.7) & 33264 (72.7) & 1.000 \\
\hline Diabetes & 4065 (35.5) & $16260(35.5)$ & 1.000 \\
\hline Dyslipidaemia & 3554 (31.1) & $14216(31.1)$ & 1.000 \\
\hline $\begin{array}{l}\text { Ischaemic heart } \\
\text { disease }\end{array}$ & 1707 (14.9) & $6118(13.4)$ & $<0.001^{*}$ \\
\hline Cerebral stroke & $5518(48.2)$ & $11390(24.9)$ & $<0.001^{*}$ \\
\hline Depression & 3231 (28.2) & $4782(10.4)$ & $<0.001^{*}$ \\
\hline COPD & $1273(11.1)$ & $4419(9.7)$ & $<0.001^{*}$ \\
\hline Asthma & 2587 (22.6) & 10229 (22.3) & 0.551 \\
\hline
\end{tabular}

${ }^{*} \chi^{2}$ test. Significance at $p<0.05$.

COPD, chronic obstructive pulmonary disease.

one wants to access the data described in this article, one could download it from the website after promising to follow the research ethics requirements.

\section{RESULTS}

The rate of asthma was not higher in dementia cases $(22.6 \%(2587 / 11442))$ than in controls $(22.3 \%$ (10 $229 / 45768$ ), table 1). The general characteristics (age, sex, income, region of residence and histories of hypertension, diabetes and dyslipidaemia) of cases were identical due to matching. Higher rates of histories of ischaemic heart disease, cerebral stroke, depression and COPD were observed in the dementia group. The adjusted OR for asthma in the dementia group was 0.97 (95\% CI 0.93 to 1.01 , $\mathrm{p}$ values $>0.05$, table 2 ) . 
Table 2 Crude and adjusted ORs (95\% Cl) of asthma in dementia participants

\begin{tabular}{|c|c|c|c|c|}
\hline \multirow[b]{2}{*}{ Characteristics } & \multicolumn{4}{|l|}{ Asthma } \\
\hline & Crude $^{*} \ddagger$ & $P$ value & 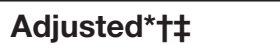 & $P$ value \\
\hline Dementia & $1.02(0.97$ to 1.07$)$ & 0.547 & 0.97 (0.92 to 1.02$)$ & 0.207 \\
\hline
\end{tabular}

*Model stratified for age, sex, income, region of residence, hypertension, diabetes and dyslipidaemia histories.

†Model adjusted for ischaemic heart disease, cerebral stroke, depression and COPD histories.

$\ddagger$ Conditional logistic regression analyses. Significance at $p<0.05$.

COPD, chronic obstructive pulmonary disease.

In the subgroup analyses, adjusted ORs for asthma were not higher in dementia cases (table 3 ). Adjusted ORs were $0.88(95 \%$ CI 0.78 to $1.00, \mathrm{p}=0.050)$ in $<80$-year-old men, 1.01 (95\% CI 0.93 to $1.09, \mathrm{p}=0.882)$ in $<80$-year-old women, 0.95 (95\% CI 0.81 to $1.12, \mathrm{p}=0.523$ ) in $\geq 80$-year-old men and 0.98 (95\% CI 0.89 to $1.09, \mathrm{p}=0.735)$ in $\geq 80$-year-old women.

\section{DISCUSSION}

Patients with dementia did not demonstrate a higher rate of previous histories of asthma than the control group in the present study. This result was consistent across all age and sex subgroups. This study was based on the older adult population considering the high prevalence of dementia in these age groups. Moreover, the potential biases between dementia and control groups were minimised by matching medical histories as well as demographic factors.

Similar to the present results, a population-based study reported a non-significant association between asthma and dementia in older adults. ${ }^{20}$ They demonstrated that asthma was related to depression $(\mathrm{OR}=2.45,95 \% \mathrm{CI} 1.06$ to 5.69) but not dementia. ${ }^{20}$ Their results might have resulted from the exclusion criteria, adjustment for the confounding effects of chronic illnesses, psychological and social factors and medication histories predisposing depression. Another population-based study using a twin cohort showed that the risk of dementia was not high in patients with asthma. ${ }^{21}$ The authors postulated that atopic disease could impact the occurrence of dementia. ${ }^{21}$ Thus, the unexpected result of no significant association between asthma and dementia was speculated due to the poor survival rates of asthma patients in their cohort. ${ }^{21}$ However, several previous studies reported a high risk of dementia in asthma patients. ${ }^{14} 15$ Although asthma could have an impact on the risk of dementia, this influence could not be significant when possible confounding effects were attenuated by matching the control group for comorbidities, as in the present study.

Table 3 Subgroup analyses of crude and adjusted ORs (95\% Cl) of asthma in dementia participants according to age and sex

\begin{tabular}{|c|c|c|c|c|}
\hline \multirow[b]{2}{*}{ Characteristics } & \multicolumn{4}{|l|}{ Asthma } \\
\hline & Crude $^{\star} \ddagger$ & $P$ value & Adjusted*†‡ & $P$ value \\
\hline \multicolumn{5}{|c|}{ Age <80-year-old men $(n=12455)$} \\
\hline Control & 1.00 & & 1.00 & \\
\hline \multicolumn{5}{|c|}{ Age <80-year-old women $(n=23410)$} \\
\hline Control & 1.00 & & 1.00 & \\
\hline \multicolumn{5}{|c|}{ Age $\geq 80$-year-old men $(n=5860)$} \\
\hline Dementia & $0.98(0.85$ to 1.14$)$ & 0.818 & 0.95 (0.81 to 1.12$)$ & 0.523 \\
\hline Control & 1.00 & & 1.00 & \\
\hline \multicolumn{5}{|c|}{ Age $\geq 80$-year-old women $(n=15485)$} \\
\hline
\end{tabular}

*Model stratified for age, sex, income, region of residence, hypertension, diabetes and dyslipidaemia histories. †Model adjusted for ischaemic heart disease, cerebral stroke, depression and COPD histories. $\ddagger$ Conditional logistic regression analyses. Significance at $p<0.05$.

COPD, chronic obstructive pulmonary disease. 
The effects of other pulmonary diseases such as COPD on the risk of dementia have been mixed in the relation between asthma and dementia in previous studies. A number of studies reported an elevated risk of dementia in patients with COPD. ${ }^{22}{ }^{23}$ The hypoxaemia due to deteriorated pulmonary function was suggested to accentuate cognitive dysfunction. ${ }^{23}$ Therefore, general pulmonary problems, rather than asthma-specific factors, could considerably contribute to the risk of dementia. Indeed, overall pulmonary disease, but not asthma alone, were related to the elevated risk of dementia in a previous study. ${ }^{24}$ A population cohort study demonstrated that COPD or combined COPD and asthma groups had a higher risk of dementia but not the asthma-only group ( $\mathrm{HR}=1.85,95 \% \mathrm{CI} 1.05$ to 3.28 for COPD and $\mathrm{HR}=1.94$, $95 \% \mathrm{CI} 1.16$ to 3.27 for combined asthma and COPD groups) ${ }^{24}$ However, the current study adjusted for COPD, thus minimising the confounding effects of COPD in assessing the association between asthma and dementia.

In addition to the confounding effects of other respiratory diseases, the influence of cardiovascular diseases and other unadjusted inflammatory or immune disorders could have mediated the association between asthma and dementia in prior studies. A number of researchers have reported that asthma is associated with inflammatory conditions in addition to those in the airway. ${ }^{2526}$ Asthma was related to the elevated risk of the proinflammatory conditions coronary heart disease and diabetes $(\mathrm{HR}=1.47$, 95\% CI 1.05 to $2.06, \mathrm{p}=0.02$ for coronary heart disease and $\mathrm{HR}=2.11,95 \%$ CI 1.43 to $3.13, \mathrm{p}<0.001$ for diabetes) ${ }^{25}$ Likewise, multiple inflammatory processes were reported to accelerate neurodegenerative changes and dementia. ${ }^{27}$ To exclude the impact of these confounding factors of cardiovascular comorbidities, the matching of the control group, in addition to the adjustments with multivariable analysis, might be effective. ${ }^{28}$

Heterogeneous biological and phenotypic features of adult asthma could mitigate the impact of asthma on dementia in this study. Although early-onset asthma is mainly related to atopic responses, late-onset asthma includes a considerable portion of non-atopic asthma and has various pathophysiologies associated with the different endotypes of asthma. ${ }^{29}$ Thus, the impact of asthma on dementia via atopy could have been attenuated in older adults. Atopy has been presumed to increase the risk of dementia probably by elevating the inflammatory burden. ${ }^{21}$ Atopic patients with asthma, eczema and rhinitis showed a 1.16-fold higher risk of dementia (95\% CI 1.01 to 1.33$).{ }^{21}$ However, asthma alone, relative to the control group, did not result in a higher risk of dementia. ${ }^{21}$

The large, representative nature of the population examined here potentiated the fidelity of the analysed results of the present study. The age of the study population was restricted to the relevant age groups of $\geq 60$ years old to minimise the early-onset dementia population. Earlyonset dementia, which occurs in the $<65$-year-old population, is different from late-onset dementia with regard to genetics, underlying pathology and relation to cardiovascular or metabolic disorders. ${ }^{30}$ Therefore, the exclusion of this younger population was important to prevent the interference of specific early-onset dementia cases with the true association between asthma and dementia. We additionally analysed the association between asthma and dementia in a $\geq 40$-year-old population. The ORs of asthma in patients with dementia were not high in this age group (online supplementary table S1). In addition, socioeconomic factors were matched between the dementia and control groups in this study. Because this study used a health insurance database, the conditions for the use of medical care, which is largely determined by socioeconomic factors, should be comparable between the dementia and control groups. In addition to socioeconomic factors, other demographic and medical histories were matched between the dementia and control groups in the present study. To evaluate the impact of matching variables on the relation between asthma and dementia, we additionally analysed the OR of asthma in dementia cases using a control group matched for age and sex (online supplementary table S2). The results also indicated no high OR of asthma in patients with dementia. The methods of classification of both asthma and dementia in our study were verified in prior studies ${ }^{16}$ (online supplementary S3Description). However, a few limitations exist, mainly due to the lack of detailed medical information in the NHIS database. The subtypes, severity and treatment of each disease could not be assessed. Subclinical or untreated dementia or asthma was not considered in this study. Although several confounders were matched or adjusted for in this study, there are still a number of possible unmeasured confounding factors, including smoking, alcohol consumption and obesity. For the matched comorbidities, the matching of comorbidities could be a negative bias on the association of asthma with dementia in this study because the timing of comorbidities was distributed throughout the follow-up period. In addition, a healthy survival effect was possible because the asthma patients who died before the occurrence of dementia were excluded from this study.

\section{CONCLUSION}

Asthma was not related to the elevated risk of neurodegenerative dementia in this older adult population.

Contributors HGC designed the study; CM and DJO analysed the data; SYK and HGC drafted and revised the paper; all authors approved the final version of the manuscript.

Funding This research was supported by funding from the National Research Foundation (NRF) of Korea (NRF-2018-R1D1A1A02085328 and NRF-2017R1C1B1007696).

Competing interests None declared.

Patient consent for publication Not required.

Provenance and peer review Not commissioned; externally peer reviewed.

Data availability statement Data may be obtained from a third party and are not publicly available. 
Open access This is an open access article distributed in accordance with the Creative Commons Attribution Non Commercial (CC BY-NC 4.0) license, which permits others to distribute, remix, adapt, build upon this work non-commercially, and license their derivative works on different terms, provided the original work is properly cited, appropriate credit is given, any changes made indicated, and the use is non-commercial. See: http://creativecommons.org/licenses/by-nc/4.0/.

\section{REFERENCES}

1 Ahmadi-Abhari S, Guzman-Castillo M, Bandosz P, et al. Temporal trend in dementia incidence since 2002 and projections for prevalence in England and Wales to 2040: modelling study. BMJ 2017;358:j2856.

2 Cho MJ, Lee JY, Kim B-S, et al. Prevalence of the major menta disorders among the Korean elderly. J Korean Med Sci 2011;26:1-10.

3 Liang X, Shan Y, Ding D, et al. Hypertension and high blood pressure are associated with dementia among Chinese Dwelling elderly: the Shanghai aging study. Front Neurol 2018;9:664.

4 Kim Y-K, Nam KI, Song J. The Glymphatic system in diabetesinduced dementia. Front Neurol 2018;9:867.

5 Bennett S, Thomas AJ. Depression and dementia: cause, consequence or coincidence? Maturitas 2014;79:184-90.

6 Deckers K, Schievink SHJ, Rodriquez MMF, et al. Coronary heart disease and risk for cognitive impairment or dementia: systematic review and meta-analysis. PLoS One 2017;12:e0184244.

7 Sears MR. Trends in the prevalence of asthma. Chest 2014;145:219-25.

8 Shin J-Y, Sohn K-H, Shin JE, et al. Changing patterns of adult asthma incidence: results from the National health insurance ServiceNational sample cohort (NHIS-NSC) database in Korea. Sci Rep 2018;8:15052.

9 Svenningsen S, Nair P. Asthma Endotypes and an overview of targeted therapy for asthma. Front Med 2017;4.

10 Christiansen SC, Schatz M, Yang S-J, et al. Hypertension and asthma: a comorbid relationship. J Allergy Clin Immunol Pract 2016;4:76-81.

11 Perez MK, Piedimonte G. Metabolic asthma: is there a link between obesity, diabetes, and asthma? Immunol Allergy Clin North Am 2014;34:777-84

12 Wang L, Gao S, Yu M, et al. Association of asthma with coronary heart disease: a meta analysis of 11 trials. PLoS One 2017;12:e0179335

13 Gao Y-H, Zhao H-S, Zhang F-R, et al. The relationship between depression and asthma: a meta-analysis of prospective studies. PLoS One 2015;10:e0132424.

14 Chen M-H, Li C-T, Tsai C-F, et al. Risk of dementia among patients with asthma: a nationwide longitudinal study. J Am Med Dir Assoc 2014;15:763-7.
15 Peng Y-H, Wu B-R, Su C-H, et al. Adult asthma increases dementia risk: a nationwide cohort study. J Epidemiol Community Health 2015;69:123-8.

$16 \mathrm{Kim} \mathrm{S}$, Kim J, Kim K, et al. Healthcare use and prescription patterns associated with adult asthma in Korea: analysis of the $\mathrm{NHI}$ claims database. Allergy 2013;68:1435-42.

17 The Korea Academy of tuberculosis and respiratory diseases. clinical practice guideline, 2018. Available: https://www.lungkorea.org/bbs/? code $=$ guide

$18 \mathrm{Kim}$ SY, Kim H-J, Lim H, et al. Bidirectional association between gastroesophageal reflux disease and depression: two different nested case-control studies using a national sample cohort. Sci Rep 2018;8:11748.

19 Kim SY, Lim J-S, Kong IG, et al. Hearing impairment and the risk of neurodegenerative dementia: a longitudinal follow-up study using a national sample cohort. Sci Rep 2018;8:15266

$20 \mathrm{Ng} \mathrm{T-P,} \mathrm{Chiam} \mathrm{P-C,} \mathrm{Kua} \mathrm{E-H.} \mathrm{Mental} \mathrm{disorders} \mathrm{and} \mathrm{asthma} \mathrm{in}$ the elderly: a population-based study. Int J Geriatr Psychiatry 2007;22:668-74.

21 Eriksson UK, Gatz M, Dickman PW, et al. Asthma, eczema, rhinitis and the risk for dementia. Dement Geriatr Cogn Disord 2008;25:148-56.

22 Tondo G, De Marchi F. Terazzi EET al. chronic obstructive pulmonary disease may complicate Alzheimer's disease: a comorbidity problem. Neurol Sci 2018.

23 Kakkera K, Padala KP, Kodali M, et al. Association of chronic obstructive pulmonary disease with mild cognitive impairment and dementia. Curr Opin Pulm Med 2018;24:173-8.

24 Rusanen M, Ngandu T, Laatikainen T, et al. Chronic obstructive pulmonary disease and asthma and the risk of mild cognitive impairment and dementia: a population based CAIDE study. Curr Alzheimer Res 2013;10:549-55.

25 Yun HD, Knoebel E, Fenta Y, et al. Asthma and proinflammatory conditions: a population-based retrospective matched cohort study. Mayo Clin Proc 2012;87:953-60.

26 Fenta YA, Tello N, Jung JA, et al. Inflammatory bowel disease and asthma: a population-based, case-control study. Inflamm Bowel Dis 2010;16:1957-62.

27 Newcombe EA, Camats-Perna J, Silva ML, et al. Inflammation: the link between comorbidities, genetics, and Alzheimer's disease. J Neuroinflammation 2018;15:276.

$28 \mathrm{Kim}$ SY, Sim S, Kim H-J, et al. Sudden sensory neural hearing loss is not predictive of myocardial infarction: a longitudinal follow-up study using a national sample cohort. Sci Rep 2018;8:946.

29 Hirano T, Matsunaga K. Late-Onset asthma: current perspectives. J Asthma Allergy 2018;11:19-27.

30 Mendez MF. Early-Onset Alzheimer disease. Neurol Clin 2017;35:263-81. 